\title{
Trends in Knee and Hip Arthroplasty in Chile Between 2004 and 2019
}

Maximiliano Barahona ${ }^{1}$, Cristian Barrientos ${ }^{1}$, Francisco Escobar Sr. ${ }^{1}$, Nicolas Diaz ${ }^{1}$, Daniel Palma Sr. ${ }^{1}$, Macarena A. Barahona ${ }^{2}$, Alvaro Martinez Sr. ${ }^{2}$, Carlos A. Infante ${ }^{1}$

1. Orthopaedic Department, Hospital Clinico Universidad De Chile, Santiago, CHL 2. Orthopaedic Department, Hospital Clinico Universidad de Chile, Santiago, CHL

Corresponding author: Maximiliano Barahona, maxbarahonavasquez@gmail.com

\section{Abstract}

\section{Introduction}

The purpose of this study is to describe the incidence rate (IR) per 100,000 inhabitants of arthroplasty in Chile between 2004 and 2019, emphasizing knee and hip arthroplasty.

\section{Methods}

This is a cross-sectional study. Patients who underwent arthroplasty between 2004 and 2019 were identified in the free access database of the Chilean Department of Statistics and Health Information (DEIS), which depends directly on the Ministry of Health. This register stores all hospital discharges of the country from private or public health centers. The trend during the period of study was analyzed using Spearman's correlation.

\section{Results}

From a total of 111,303 patients, 133,518 arthroplasties were performed. Hip arthroplasty (HA) accounted for $73.35 \%$, followed by knee arthroplasty (KA) $(23,92 \%)$. A significant upward trend was found in HA $(r h o=0.95, p<0.000)$ and KA $(r h o=0.98, p<0.000)$. Most of the surgeries were done within the Public Health Network (61,6\%), but $20 \%$ of patients affiliated with public insurance underwent arthroplasty in a private center. Patients above 60 years of age affiliated with private insurance underwent $1.8 \mathrm{HA}$ and $2.5 \mathrm{KA}$ for every one HA and KA undergone by patients of the same age group who were affiliated with public insurance.

\section{Conclusion}

HA was more frequent than KA. A significant gap was found in the incidence of arthroplasty as compared to countries belonging to the Organization for Cooperation and Economic Development, given by a less aged population and by inequity in health access. Wider coverage and a national registry for arthroplasty must be considered in Chilean health policies.

Review began 12/04/2020 Review ended 12/13/2020 Published 12/20/2020

\section{() Copyright 2020}

Barahona et al. This is an open access article distributed under the terms of the Creative Commons Attribution License CC-BY 4.0., which permits unrestricted use, distribution, and reproduction in any medium, provided the original author and source are credited.
Categories: Orthopedics, Public Health, Epidemiology/Public Health

Keywords: knee arthroplasty, hip arthroplasty, national registries, arthroplasty, epidemiology

\section{Introduction}

There are 40 arthroplasty registries worldwide, including countries of Europe, North America, Africa, Asia, and Oceania. In contrast, Chile, along with other Latin American countries, have not developed registries. Brazil is the only one that has reported a project to be implemented in the near future [1].

A recent study carried out by the Organization for Cooperation and Economic Development (OECD) estimates that the number of hip arthroplasties within the next 35 years will grow $100 \%$, overstressing health systems [2]. National registries offer the potential to better outcomes and identify, implement, and share best practices. The effective use of quality registries can lead to better health outcomes at a lower cost for society [3].

In the Chilean health system, population coverage is provided mainly by two insurances: the public by the National Health Found (FONASA) and the private by the Health Insurance Institutions (ISAPRE). During the period studied, $72.5 \%$ of the Chilean population was affiliated with FONASA; meanwhile, $17.4 \%$ was affiliated with ISAPRE.

Two types of institutions provide inpatients services: the public health network (PHN) and private health institutions (PHI). PHN attends patients affiliated with FONASA. PHI corresponds to a broad spectrum that includes university hospitals, workers' mutuals, armed forces hospitals, police hospitals, and centers 
belonging to investment funds. Some patients affiliated with FONASA choose treatment in PHI at their expense to avoid the wait time for surgery.

The purpose of this study is to describe the incidence rate per 100,000 inhabitants (IR) of arthroplasty in Chile, emphasizing knee and hip arthroplasty. The hypothesis is that hip and knee arthroplasty have an upward trend, the IR of hip arthroplasty being higher than knee arthroplasty.

\section{Materials And Methods}

This is a cross-sectional study. Patients who underwent arthroplasty between 2004 and 2019 were identified in the free access database of the Chilean Department of Statistics and Health Information (DEIS), which depends directly on the Ministry of Health. This register stores all hospital discharges of the country, both from PHN and PHI. During the analysis of the database, inconsistencies were found in the years 2006 and 2010 to 2012. This was reported to DEIS via the National Transparency Portal in April 2020. The last database updates of each of those years were used. Registres from 2006 were updated on August 11, 2020, and the ones between 2010 and 2012 on May 22, 2020 (Figure 1).

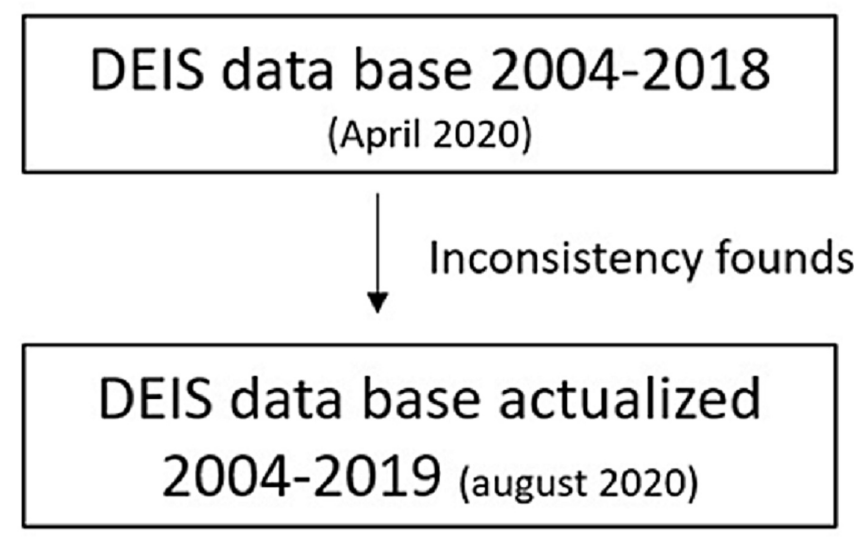

Data downloaded.

\section{$26,358,740$ hospital discharges 2004-2019}

Identification using national code system for surgery in ACCESS (Microsoft ${ }^{\circledR}$ ).

$$
\begin{aligned}
& \text { 133,518 }(0.5 \%) \\
& \text { arthroplasties. } \\
& \text { Data exported to Stata v15 } \\
& \text { for analysis. }
\end{aligned}
$$

FIGURE 1: Flowchart

DEIS: Department of Statistics and Health Information 
The databases from 2004 to 2019 were downloaded from the DEIS homepage https://deis.minsal.cl/\#datosabiertos. The software program Microsoft Access (Microsoft Corporation, Redmond, Washington) was used to manage the data. Patients were identified using the national code for surgeries: hip arthroplasty $(\mathrm{HA})=2104128,21041292104228$ and 2104229; knee arthroplasty $(\mathrm{KA})=$ 2104153; shoulder arthroplasty $(\mathrm{SA})=2104042$ and 2501044; elbow arthroplasty $(\mathrm{EA})=2104062$; wrist arthroplasty $(\mathrm{WA})=2104179$; ankle arthroplasty $(\mathrm{AA})=2104174$. Data were exported to STATA v.15 (StataCorp LP, College Station, Texas) for statistical analysis.

The incidence rate (IR) was calculated per 100,000 inhabitants using the overall population published in the annual report of the National Statistics Institute of Chile (INE) [4]. From the same report, the number of men and women was used to calculate the IR per gender. The proportion of patients affiliated with public insurance (FONASA) or private insurance (ISAPRE) was obtained from the annual report of FONASA and was used to calculate the IR per health coverage. Additionally, in each health insurance, the IR of patients older than 60 years of age - who are at a higher risk of needing an arthroplasty - was calculated.

The data has the primary diagnosis coded according to the International Classification of Diseases, 10th revision (ICD-10). Patients undergoing arthroplasty due to fractures were identified according to the ICD-10 classification. It was not possible to recognize if the patient underwent unicompartmental, patellofemoral, or total knee replacement, as all those procedures have the code 2014153. Also, no different code is used to identify if the prosthesis was constrained or unconstrained, primary, or revision. In the case of hip replacement, it was possible to detect total and hemiarthroplasty, as they have different codes. The revision rate was estimated, including all patients who had more than two arthroplasties in the same joint and patients diagnosed with mechanical complications or infections.

Spearman's correlation was used to analyze the trend in the period studied; a significance of 0.05 was used. If the trend was significant, a linear regression was estimated to predict the IR in 2030. The r-squared (R2) and parameters $\beta 0$ and $\beta 1$ are reported. After every linear regression, heteroscedasticity and the normal distribution of the residuals were evaluated.

\section{Results}

A total of 133,518 arthroplasties were found, which represents 0,5\% of the hospital discharges between 2004 and 2019; the PHN managed 82,287 (61.6\%) surgeries. Procedures in patients affiliated with FONASA were 97,065 (72.60\%), and in patients affiliated with ISAPRE were 23,948 (17.9\%). The number of patients included was 111,303 , with a mean age of 67 years $( \pm 14.44)$, and $72360(65.01 \%)$ were female.

The most frequent arthroplasty was HA 97,931 (73.35\%), followed by KA 31,943 (23,92\%), SE 2,735 (2.05\%) AA 369 (0.28\%), WA 284 (0.21\%), and EA 256 (0.19\%). The IR of each arthroplasty is shown in Table 1. 


\section{Cureus}

\begin{tabular}{|c|c|c|c|c|c|c|}
\hline Year & HA & KA & sa & ea & aa & wa \\
\hline 2004 & 13.18 & 2.56 & 0.14 & 0 & 0.01 & 0.02 \\
\hline 2005 & 17.15 & 2.59 & 0.29 & 0.01 & 0.01 & 0.27 \\
\hline 2006 & 18.19 & 3.61 & 0.22 & 0.01 & 0 & 0.05 \\
\hline 2007 & 24.05 & 5.22 & 0.42 & 0.04 & 0.02 & 0.11 \\
\hline 2008 & 24.32 & 5.99 & 0.44 & 0.04 & 0.06 & 0.13 \\
\hline 2009 & 29.58 & 7.41 & 0.54 & 0.05 & 0.10 & 0.05 \\
\hline 2010 & 28.21 & 6.41 & 0.54 & 0.03 & 0.11 & 0.09 \\
\hline 2011 & 43.71 & 7.98 & 0.59 & 0.10 & 0.15 & 0.13 \\
\hline 2012 & 38.17 & 10.77 & 0.84 & 0.07 & 0.08 & 0.07 \\
\hline 2013 & 37.99 & 13.91 & 1.17 & 0.18 & 0.12 & 0.10 \\
\hline 2014 & 37.56 & 10.29 & 1.29 & 0.11 & 0.12 & 0.09 \\
\hline 2015 & 42.39 & 14.33 & 1.30 & 0.12 & 0.07 & 0.09 \\
\hline 2016 & 45.50 & 16.59 & 1.61 & 0.13 & 0.27 & 0.17 \\
\hline 2017 & 50.78 & 18.84 & 1.86 & 0.11 & 0.44 & 0.10 \\
\hline 2018 & 49.58 & 23.84 & 2.01 & 0.22 & 0.24 & 0.08 \\
\hline 2019 & 54.49 & 28.23 & 2.01 & 0.20 & 0.24 & 0.14 \\
\hline
\end{tabular}

\section{TABLE 1: Rate of arthroplasty per 100,000 inhabitants per year}

Abbreviations: HA: hip arthroplasty; KA: knee arthroplasty; SA: shoulder arthroplasty; EA: elbow arthroplasty; AA: ankle arthroplasty; WA: wrist arthroplasty

\section{Hip arthroplasty}

A total of 97,931 HA were found; of them, 63,323 (64.66\%) were performed in the PHN. The median age was 67 years $( \pm 15)$ and $41,325(42.20 \%)$ patients were under 65 years of age. Procedures on patients affiliated with FONASA were 73,307 (74.86\%); meanwhile, 16,249 (15.59\%) were affiliated with ISAPRE. In 2004, the IR of HA was 13.18, which increased to 54.49 in 2019. This upward trend was significant $($ rho $=0.95, \mathrm{p}<0.000)$ (Table 2). 


\section{Cureus}

\begin{tabular}{|c|c|c|c|c|c|c|}
\hline Year & $H A^{*}$ & $\mathrm{THA}^{*}$ & Fracture* & PHA $^{*}$ & HHA/Fracture & Fracture/THA \\
\hline 2004 & 13.18 & 9.755 & 5.64 & 3.42 & 0.607 & 0.427 \\
\hline 2005 & 17.15 & 12.97 & 7.11 & 4.18 & 0.587 & 0.414 \\
\hline 2006 & 18.19 & 13.34 & 7.03 & 4.84 & 0.688 & 0.386 \\
\hline 2007 & 24.05 & 19.13 & 8.25 & 4.91 & 0.595 & 0.343 \\
\hline 2008 & 24.32 & 19.84 & 8.52 & 4.47 & 0.525 & 0.350 \\
\hline 2009 & 29.58 & 25.15 & 9.53 & 4.42 & 0.463 & 0.322 \\
\hline 2010 & 28.21 & 23.77 & 9.79 & 4.43 & 0.453 & 0.347 \\
\hline 2011 & 43.71 & 38.64 & 10.14 & 5.07 & 0.499 & 0.232 \\
\hline 2012 & 38.17 & 33.01 & 10.91 & 5.15 & 0.472 & 0.285 \\
\hline 2013 & 37.99 & 33.34 & 10.83 & 4.64 & 0.428 & 0.285 \\
\hline 2014 & 37.56 & 33.09 & 11.06 & 4.46 & 0.403 & 0.294 \\
\hline 2015 & 42.39 & 37.41 & 12.04 & 4.97 & 0.413 & 0.284 \\
\hline 2016 & 45.50 & 40.59 & 12.68 & 4.90 & 0.387 & 0.278 \\
\hline 2017 & 50.78 & 46.06 & 13.47 & 4.72 & 0.350 & 0.265 \\
\hline 2018 & 49.58 & 45.29 & 12.59 & 4.29 & 0.340 & 0.253 \\
\hline 2019 & 54.49 & 50.20 & 13.16 & 4.29 & 0.326 & 0.246 \\
\hline
\end{tabular}

\section{TABLE 2: Summary of the findings of hip arthroplasty}

*Per 100,000 inhabitants

Abbreviations: HA: hip arthroplasty; THA: total hip arthroplasty; fracture: hip replacement due to femoral neck fracture; HHA: hip hemiarthroplasty; PHA: partial hip arthroplasty

Regarding total hip arthroplasty (THA), the IR in 2004 was 9.75; meanwhile, it was 50.20 in 2019 (Table 1). This upward trend was significant $(\mathrm{rho}=0.96, \mathrm{p}<0.000)$ and follows a linear model $(\mathrm{R} 2=0.94)$ of parameters $\beta 0=-5192.878(p<0.00) \beta 1=2.597(p<0.00)$, which predicts an IR of 78.14 in 2030 (Figure 2). 


\section{Cureus}

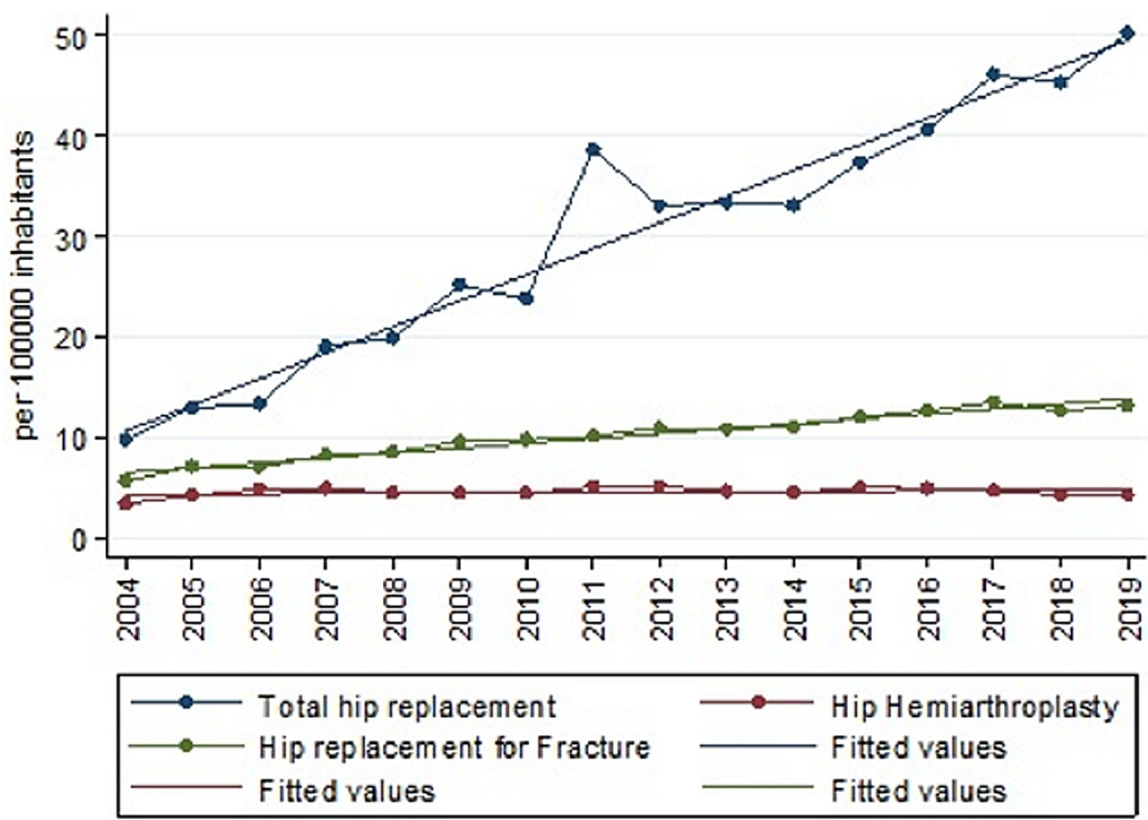

FIGURE 2: The trend of procedures per 100,000 inhabitants between 2004 and 2019 in THA, PHA, and HA due to intracapsular fractures

Abbreviations: HA: hip arthroplasty; THA: total hip arthroplasty; PHA: partial hip arthroplasty

In 2004, the IR in female patients was 12.86, which increased to 62.87 in 2019. This trend was significant (rho=0.9676, p 00.000). Male IR was 37.0 in 2019 (Figure 3) and had a significant upward trend (rho=0.9618, $\mathrm{p}<0.000)$. The proportion of males undergoing THA has slightly increased, reaching statistical significance (rho=0.65, p=0.0064) (Table 3).

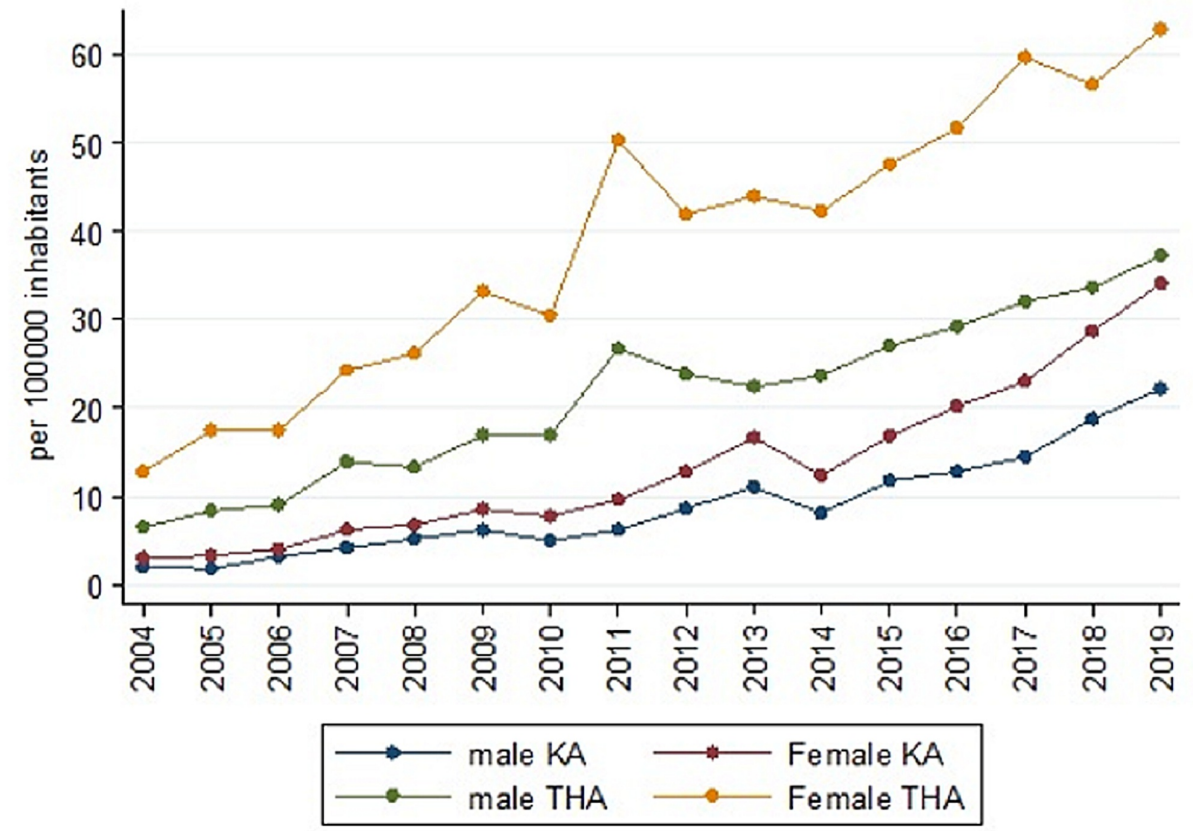

FIGURE 3: Trend of procedures by gender per 100,000 inhabitants between 2004 and 2019

Abbreviations: KA: knee arthroplasty; THA: total hip arthroplasty 


\section{Cureus}

\begin{tabular}{|c|c|c|c|c|c|c|}
\hline Year & Male KA* & Female KA* & Male THA* & Female THA* & Male/KA & Male/THA \\
\hline 2004 & 2.04 & 3.06 & 6.58 & 12.86 & 0.399 & 0.338 \\
\hline 2005 & 1.89 & 3.27 & 8.40 & 17.44 & 0.367 & 0.325 \\
\hline 2006 & 3.16 & 4.05 & 9.05 & 17.56 & 0.438 & 0.340 \\
\hline 2007 & 4.23 & 6.20 & 13.88 & 24.28 & 0.405 & 0.363 \\
\hline 2008 & 5.19 & 6.77 & 13.35 & 26.21 & 0.433 & 0.337 \\
\hline 2009 & 6.24 & 8.56 & 16.97 & 33.18 & 0.421 & 0.338 \\
\hline 2010 & 5.01 & 7.78 & 16.90 & 30.51 & 0.391 & 0.356 \\
\hline 2011 & 6.24 & 9.68 & 26.71 & 50.33 & 0.391 & 0.346 \\
\hline 2012 & 8.65 & 12.84 & 23.87 & 41.97 & 0.402 & 0.362 \\
\hline 2013 & 11.09 & 16.68 & 22.50 & 43.97 & 0.399 & 0.338 \\
\hline 2014 & 8.13 & 12.42 & 23.68 & 42.30 & 0.395 & 0.358 \\
\hline 2015 & 11.72 & 16.88 & 27.06 & 47.55 & 0.409 & 0.362 \\
\hline 2016 & 12.87 & 20.23 & 29.27 & 51.68 & 0.388 & 0.361 \\
\hline 2017 & 14.51 & 23.07 & 32.13 & 59.71 & 0.385 & 0.349 \\
\hline 2018 & 18.82 & 28.74 & 33.70 & 56.66 & 0.395 & 0.372 \\
\hline 2019 & 22.24 & 34.10 & 37.26 & 62.87 & 0.394 & 0.372 \\
\hline
\end{tabular}

TABLE 3: Summary by gender of the rate by 100,000 inhabitants of knee and hip arthroplasty

*Per 100,000 inhabitants

Abbreviations: KA: knee arthroplasty; THA: total hip arthroplasty

The IR of patients affiliated with FONASA who underwent THA was 9.62 in 2004; meanwhile, it was 49.16 in 2019. However, the IR of patients affiliated with ISAPRE increased to 52.07 in 2019 from 10.4 in 2004 (Figure 4). Between 2009 and 2018, IR in patients above 60 years was 142.25 and 259.29 for FONASA and ISAPRE, respectively - which represents $1.8 \mathrm{HA}$ in patients over 60 years of age affiliated to ISAPRE for every $1 \mathrm{HA}$ of patients affiliated to FONASA over 60 years of age. The proportion of THA performed in PHN increased from 0.57 in 2004 to 0.68 in 2011 (Figure 5); then, it went down to 0.62 in 2019 (rho -0.144, p=0.5944) (Table 4). 


\section{Cureus}

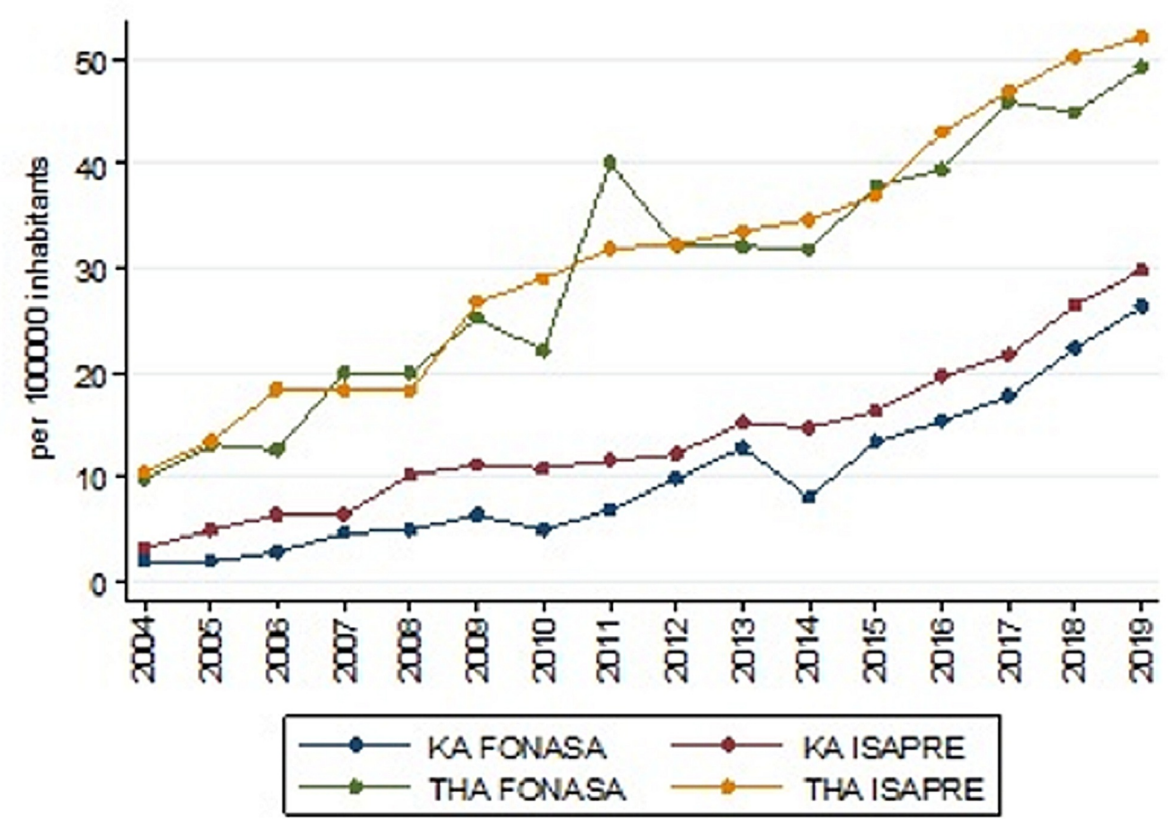

FIGURE 4: Trend in HA and KA by type of health insurance

Abbreviations: KA: knee arthroplasty; HA: hip arthroplasty

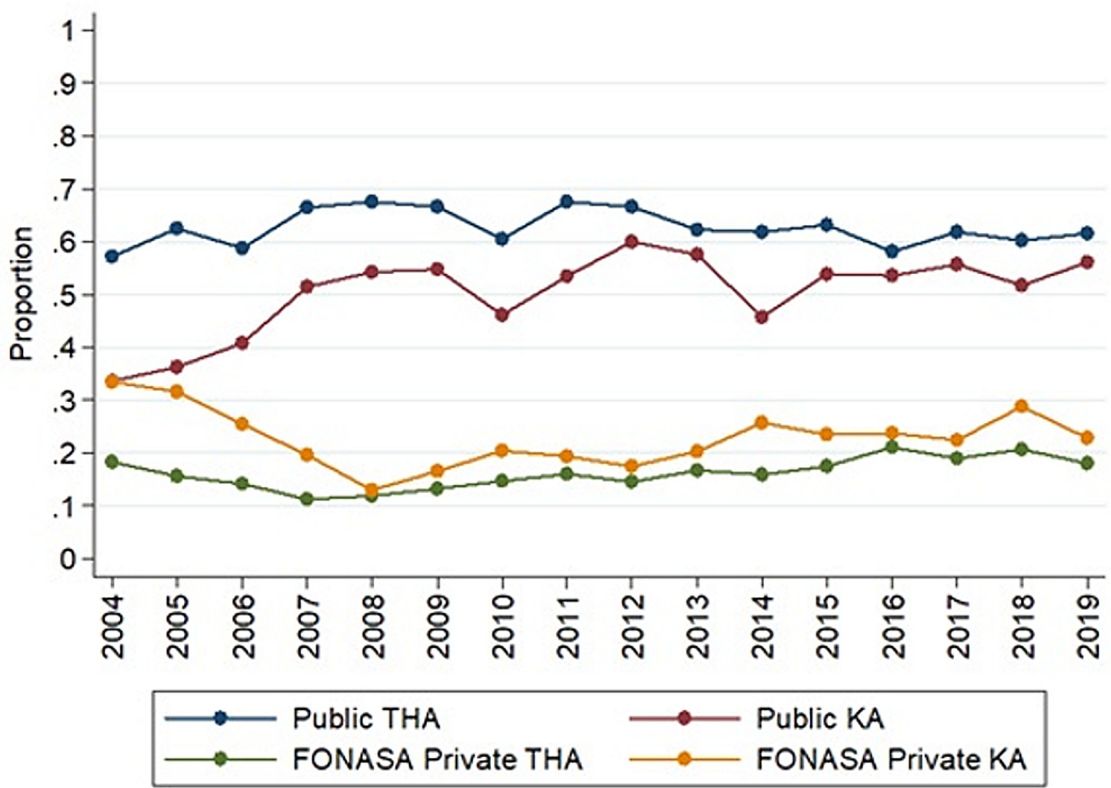

FIGURE 5: The proportion of patients that underwent HA and KA in a public health center and the rate of patients affiliated to the public insurance (FONASA) that underwent HA and KA in a private health center.

Abbreviations: KA: knee arthroplasty; HA: hip arthroplasty 


\begin{tabular}{|llllllllll|}
\hline Year & F KA* & I KA & F THA & I THA & PHN/HA & PHN/KA & F PHI KA & F PHI THA \\
\hline 2004 & 1.87 & 3.14 & 9.62 & 10.40 & 0.571 & 0.336 & 0.334 & 0.182 \\
\hline 2005 & 1.86 & 4.86 & 13.05 & 13.42 & 0.625 & 0.362 & 0.315 & 0.155 \\
2006 & 2.71 & 6.34 & 12.54 & 18.33 & 0.587 & 0.408 & 0.254 & 0.141 \\
2007 & 4.58 & 6.34 & 19.91 & 18.28 & 0.665 & 0.514 & 0.196 & 0.112 \\
2008 & 4.85 & 10.11 & 19.94 & 18.15 & 0.675 & 0.542 & 0.130 & 0.118 \\
2009 & 6.32 & 11.11 & 25.20 & 26.70 & 0.666 & 0.547 & 0.165 & 0.132 \\
2010 & 4.84 & 10.75 & 22.06 & 28.94 & 0.604 & 0.461 & 0.203 & 0.146 \\
2011 & 6.79 & 11.52 & 40.05 & 31.85 & 0.675 & 0.533 & 0.192 & 0.160 \\
2012 & 9.76 & 12.18 & 32.11 & 32.19 & 0.665 & 0.599 & 0.174 & 0.145 \\
2013 & 12.80 & 15.10 & 32.04 & 33.52 & 0.622 & 0.575 & 0.202 & 0.166 \\
2014 & 8.022 & 14.68 & 31.69 & 34.64 & 0.618 & 0.456 & 0.257 & 0.158 \\
2015 & 13.34 & 16.24 & 37.78 & 36.89 & 0.631 & 0.538 & 0.234 & 0.174 \\
2016 & 15.27 & 19.57 & 39.40 & 43.00 & 0.580 & 0.535 & 0.237 & 0.211 \\
2017 & 17.70 & 21.62 & 45.95 & 46.93 & 0.618 & 0.556 & 0.224 & 0.189 \\
2018 & 22.28 & 26.47 & 44.87 & 50.24 & 0.602 & 0.516 & 0.287 & 0.207 \\
2019 & 26.28 & 29.76 & 49.16 & 52.07 & 0.615 & 0.560 & 0.228 & 0.180 \\
\hline & & & & & & & & \\
\hline
\end{tabular}

TABLE 4: Summary of KA and THA per health insurance and type of institution

*Per 100,000 inhabitants

Abbreviations: F: FONASA (public insurance); KA: knee arthroplasty; I: ISAPRE (private insurance); THA: total hip arthroplasty; PHN: public health network; PHI: private health institutions

In 2004, $42.78 \%$ of HA were performed due to hip fracture; meanwhile, in 2019, hip fracture caused $24.16 \%$ of the performed HA. This downward trend was significant $(\mathrm{rho}=-0.87, \mathrm{p}<0.000)$ (Table 1). Of intracapsular fractures, $60.75 \%$ were treated with hemiarthroplasty (HHA) in 2004; in contrast, it was only $32.60 \%$ in 2019. This downward trend was significant $(\mathrm{rho}=-0.9588, \mathrm{p}<0.000)$.

Hip revision procedures in 2004 were estimated to be 84 (0.05\%); meanwhile, it went up to 341 in 2019 $(0.04 \%)$. This upward trend was significant $(\mathrm{rho}=0.96, \mathrm{p}<0.000)$ and follows a linear model $(\mathrm{R} 2=0.91)$ of parameters $\beta 0=-35059.66(p<0.00)$ and $\beta 1=17.53(p<0.00)$, which predict a total of 525 revisions in 2030 . The proportion of revision estimated has remained constant $(\mathrm{rho}=-0.34, \mathrm{p}=0.1959)$.

\section{Knee arthroplasty}

A total of 31,943 KA were performed; of them, 17,004 (53.23\%) were carried out in the PHN. The median age was 66 years $( \pm 11)$ and 13,512 patients $(42.30 \%)$ were under 65 years old. Procedures on patients affiliated to FONASA were 21,305 (66.70\%); meanwhile, 6,961 (21.79\%) were on patients affiliated to ISAPRE.

In 2004, KA IR was 2.56, which increased to 28.23 in 2019 (Table 1). This upward trend was significant $(\mathrm{rho}=0.98, \mathrm{p}<0.000)$. The trend of KA has two slopes, one from 2004 to 2014 and the other between 2014 and 2019 (Figure 6). According to this last slope, a linear regression ( $\mathrm{r} 2=0.98)$ with parameters $\beta 0=-6919.51$ $(p<0.00) \beta 1=3.44(p<0-00)$ estimates a rate of 65.14 TKA per 100,000 inhabitants in 2030. 


\section{Cureus}

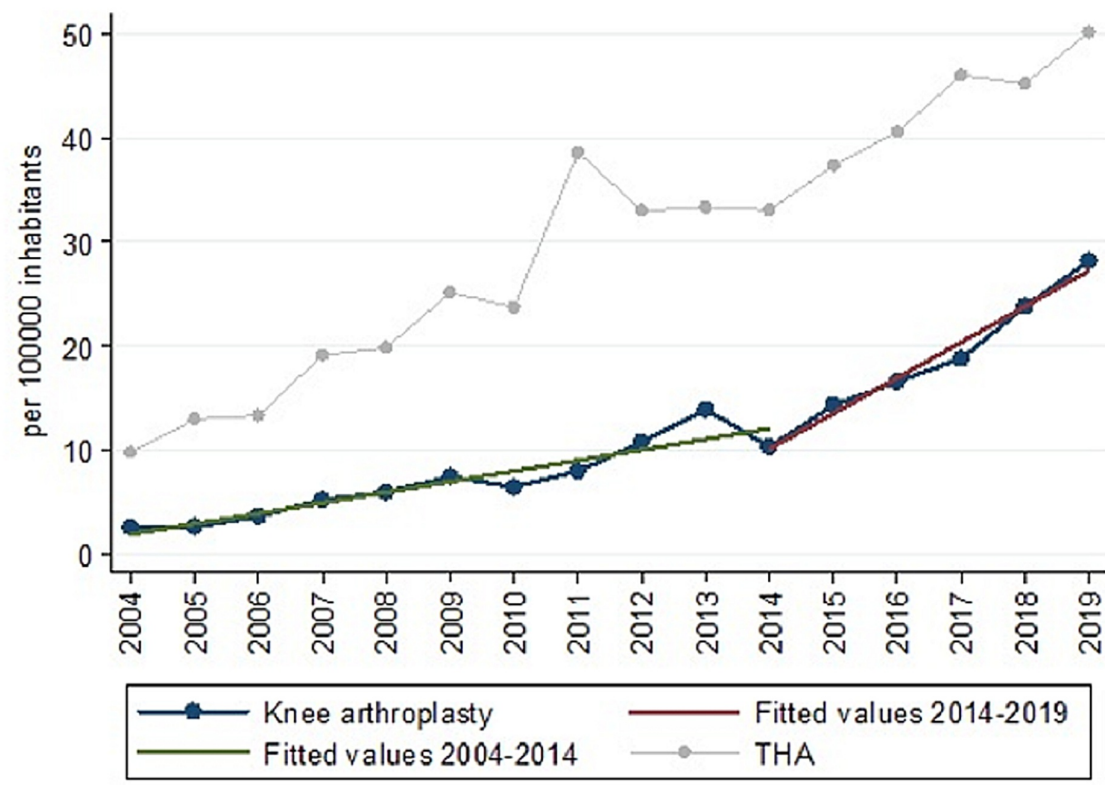

\section{FIGURE 6: The trend of procedures per 100,000 inhabitants between 2004 and 2019 in KA}

Abbreviations: KA: knee arthroplasty; THA: total hip arthroplasty

Male IR was 2.04 KA in 2004, which increased to 22.24 in 2019; this trend was significant (rho=0.97, $\mathrm{p}<0.000$ ). Female IR also had a significant upward trend (rho=0.9794, p<0.000), reaching 26.28 KA in 2019. The proportion of males had remained constant ( $r h o=-0.34, \mathrm{p}=0.1919)$, from $40 \%$ in 2004 to 39\% in 2019 (Table 3).

FONASA IR was 1.87 in 2004, and it increased to 26.28 in 2019. However, the IR in patients affiliated with ISAPRE increased to 29.76 in 2019 from 3.14 in 2004 (Figure 4). Between 2009 and 2018, IR in patients above 60 years was 54.87 and 137.84 for FONASA and ISAPRE, respectively, which represents 2.5 KA in patients over 60 years of age affiliated to ISAPRE for every 1 KA of patients affiliated to FONASA over 60 years of age. The proportion of patients affiliated with FONASA was $37.3 \%$ in 2004 and $47.8 \%$ in 2019; this upward trend was statistically significant ( $r h o=0.7441, \mathrm{p}=0.0009$ ). In 2004 , the proportion of KA performed in the PHN was $33.65 \%$, and it went up to $56.04 \%$ in 2019 (Figure 3). This upward trend was significant ( $\mathrm{rho}=0.57, \mathrm{p}=0.021$ ) (Table 4).

Knee revision procedures in 2004 were estimated to be 10 (0.02\%). Meanwhile, it went up to 105 in 2019 (0.02\%). This upward trend was significant $(\mathrm{rho}=0.89, \mathrm{p}<0.000)$ and follows a linear model $(\mathrm{R} 2=0.75)$ of parameters $\beta 0=-9270.16 \beta 1=4.627$, which predict a total of 125 revisions in 2030 . The proportion of revision estimated has remained constant ( $\mathrm{rho}=-0.35, \mathrm{p}=0.2493)$.

\section{Discussion}

The main findings of this study were that HA and KA had an upward trend in the period studied and that HA was 2.5 times more frequent per 100,000 inhabitants than KA. Also, female patients underwent a higher proportion of arthroplasty, this difference being wider in hip arthroplasty.

According to a report of OECD, Chile was second last in IR for HA and KA in 2017 [5]. Between 1995 and 2000, Australia had the same population as Chile in 2019 (around 18 million). By then, Australia had an IR between 166 and $179 \mathrm{HA}$ and 181 to $196 \mathrm{KA}$, way above the 54 and 28 IR found in this study [6]. One factor that is responsible for this difference is the age of the population. According to the World Bank, Europe has $20 \%$ of their population above 65 years; Japan has 28\%, the United States (US) and Australia have 16\%; and the average of OECD countries is $16 \%$; meanwhile, Chile has $12 \%$ of its population above 65 years.

Another crucial explanation is that Chile is a country with low coverage for a core set of services and health expenditures (HE). Chile was way below the OECD average of HE estimated in 2018 (3394 USD PPD), reaching 2182 USD per person. HE was 50\% government/compulsory and the other half out of the patient's pocket [5]. Nevertheless, since 2008, Chile has continued to report annual health spending increases above 
$5 \%$, reaching in $2018,8.8 \%$ of the gross domestic product, which was above the OECD average (8.7\%). Also, HE from public sources as a share of total government expenditures in 2017 was 17\% (above OECD average 15\%) [5], which explains the significant upward trend in IR of arthroplasty.

The Chilean health system relies heavily on PHI [7]. This study reports that near $20 \%$ of patients affiliated to FONASA, the public insurance, underwent arthroplasty in a PHI. Also, the proportion of THA in PHN had varied between $58 \%$ and $68 \%$ between 2004 and 2019. Moreover, PHN had never performed above $60 \%$ of the KA, achieving the most in 2013 (59\%). In England, an upward trend between 2004 and 2013 was reported in THA in private health centers, going from $2 \%$ to $20 \%$ [8]. In Australia, approximately two-thirds of the procedures are performed in public centers [9]. Remarkably, between 2009 and 2018, patients above 60 years, who are at a higher risk of needing arthroplasty, represented $17 \%$ and $8 \%$ of the patients affiliated with ISAPRE and FONASA, respectively $[4,10]$. Adjusting by age, patients affiliated with private insurance underwent 1.8 HA and 2.5 KA for every one HA and KA undergone by patients over 60 years old affiliated with public insurance. Inequity in access is a significant issue in the Chilean health system [11-12].

Explicit Health Guarantees (GES) are a set of benefits guaranteed by the Chilean law allowing access, opportunity, financial protection, and quality of care in a designated list of diseases. In 2006, HA for patients older than 65 years was included. This is probably the main reason for the different slope found in this study between HA and KA. The 2.6 increment by year is insufficient to reach the IR of OECD countries. Moreover, the Australian and the Finnish registries show that the IR of KA has overcome the IR for HA [1314]. Regarding knee replacement, GES only covers non-surgical treatment.

The age threshold of GES limits the increase in HA. Reports from National Arthroplasty registers show an increased burden of patients requiring arthroplasty below 65 years of age [15]. Moreover, this study shows that $42 \%$ of the patients that underwent HA and KA were younger than 65 , leaving them unprotected. Osteoarthritis has the most impact on the US healthcare system due to its high prevalence and disability [16]. Therefore it does not seem cost-effective to exclude working-age patients from GES and condemning them to a waiting list for surgery; especially when THA has been considered the operation of the century due to its impact on quality of life [17]. To add younger patients to GES will allow them to get back to work early. According to unpublished data of the Ministry of Health released by the National Portal of Transparency, the waiting time for surgery between 2012 and 2019 for non-GES HA and KA was 2.19 years (interquartile range, 1.12-3.07). This time is considerably longer than reported by OECD, in which the mean waiting time for HA and KA does not exceed one year [18]. More extensive coverage for HA and the inclusion of KA must be carefully considered in Chilean health policies.

A high proportion of HA replacement has been found in this study due to hip fracture. The reported ratio varies from $2.4 \%$ in the US, $4.9 \%$ in New Zealand, $6 \%$ in Switzerland, to $9.3 \%$ in Sweden. Those countries have an IR ranging from 180 to 194 in 2017 . Hence the IR for hip fracture is between 4.7 to 16.2 , which is like the findings in Chile (13.1). This should focus Chilean efforts to gain access to patients with severe osteoarthritis, which was the main indication (78.9\% to $87.1 \%$ ) for THA in those countries [1].

The optimal treatment for femoral neck fractures in patients over 60 years continues to be debated [19]. The England registry between 2011 to 2016 shows that HHA was used in 50\% of neck fractures and THA has a downward trend. United Kingdom's guidelines demand that a hip fracture must undergo surgery in less than three days [20]. Therefore, a possible explanation is that HHA is a more familiar technique for a general trauma surgeon, not requiring to wait for a hip surgeon [21]. On the other side, this study shows a significant downward trend in HHA. No guidelines regarding timing in hip fracture are in Chile, so a more extended hospital stay has been reported [22], which is far from ideal but seems to allow patients to undergo THA. The Australian registry also indicates a low usage of THA in femoral neck fractures, reaching 23.7\%; however, the rate was higher when the surgery was performed on weekdays and by a hip surgeon [23]. On the other hand, a report from the US shows that THA for hip fracture has increased in patients below 70 years and patients with private insurance [24].

Arthroplasty nationals registries have contributed to more prolonged survival, better functional outcomes, and the identification of health centers with high rates of complications to improve their results [25]. Chile is having an upward trend in HA and KA but is far behind other OECD countries. Along with improving access, it is time to take the next step in the quality of the Chilean health system and create a national arthroplasty register, aiming to make joint replacements more cost-effective.

The limitations of this study are related to the database. The Chilean national code for surgeries urgently requires an update that allows identifying different subtypes of arthroplasty like unicompartmental knee replacement or revision surgery. Also, the database does not store any variable related to the follow-up of the patients. The strength of this report is that all the procedures performed were included, including the types of institutions or insurance, race, socioeconomic, or geographic factors.

\section{Conclusions}

Arthroplasty represents $0.5 \%$ of the hospital discharges between 2004 and 2019 in Chile. Hip and knee 
arthroplasties were by far the most frequent. Both show an upward trend during the study period with hip replacement being more frequent than knee replacement. Overall, HA was 2.5 times more frequent per 100,000 inhabitants than knee replacement. A significant gap was found in the incidence of arthroplasty with OECD countries, given the less aged population and the inequity in health access that exists in Chile. Wider coverage and a national registry for arthroplasty must be considered in Chilean health policies.

\section{Additional Information \\ Disclosures}

Human subjects: All authors have confirmed that this study did not involve human participants or tissue. Animal subjects: All authors have confirmed that this study did not involve animal subjects or tissue. Conflicts of interest: In compliance with the ICMJE uniform disclosure form, all authors declare the following: Payment/services info: All authors have declared that no financial support was received from any organization for the submitted work. Financial relationships: All authors have declared that they have no financial relationships at present or within the previous three years with any organizations that might have an interest in the submitted work. Other relationships: All authors have declared that there are no other relationships or activities that could appear to have influenced the submitted work.

\section{Acknowledgements}

The first author acknowledges the support of Leonel Barahona and Luis Pasten in the elaboration of this study.

\section{References}

1. Abdelaal MS, Restrepo C, Sharkey PF: Global perspectives on arthroplasty of hip and knee joints . Orthop Clin North Am. 2020, 51:169-176. 10.1016/j.ocl.2019.11.003

2. Pabinger C, Lothaller H, Portner N, Geissler A: Projections of hip arthroplasty in OECD countries up to 2050. Hip Int. 2018, 28:498-506. 10.1177/1120700018757940

3. Malchau H, Garellick G, Berry D, et al.: Arthroplasty implant registries over the past five decades: development, current, and future impact. J Orthop Res. 2018, 36:2319-2330. 10.1002/jor.24014

4. Compendio estadístico del 2018 elaborado por el Instituto Nacional de Estadística [Website in Spanish] . (2018). https://www.ine.cl.

5. OECD: Health at a Glance . OECD Publishing, Paris, France; 2019. 10.1787/4dd50c09-en

6. Inacio MC, Graves SE, Pratt NL, Roughead EE, Nemes S.: Increase in total joint arthroplasty projected from 2014 to 2046 in Australia: a conservative local model with international implications. Clin Orthop Relat Res. 2017, 475:2130-2137. 10.1007/s11999-017-5377-7

7. Atun R, De Andrade LOM, Almeida G, et al.: Health-system reform and universal health coverage in Latin America. Lancet. 2015, 385:1230-1247. 10.1016/S0140-6736(14)61646-9

8. Sutaria S, Kirkwood G, Pollock AM: An ecological study of NHS funded elective hip arthroplasties in England from 2003/04 to 2012/13. J R Soc Med. 2019, 112:292-303. 10.1177/0141076819851701

9. Harris I, Cuthbert A, Lorimer M, de Steiger R, Lewis P, Graves SE: Outcomes of hip and knee replacement surgery in private and public hospitals in Australia. ANZ J Surg. 2019, 89:1417-1423. 10.1111/ans.15154

10. Development CMoS (National Socioeconomic Characterization Survey) [Website in Spanish] . (2017). http://observatorio.ministeriodesarrollosocial.gob.cl/casen-multidimensional/casen/casen 2017.php.

11. Fraser B: Violent protests in Chile linked to health-care inequities . Lancet. 2019, 394:1697-1698. 10.1016/S0140-6736(19)32720-5

12. López R, Miller SJ: Chile: the unbearable burden of inequality. World Dev. 2008, 36:2679-2695. 10.1016/j.worlddev.2008.01.012

13. National Joint Replacement Registry 2017 annual report. Australian Orthopaedic Association . (2017). https://aoanjrr.sahmri.com/documents/10180/397736/Hip\%2C\%20Knee\%20\%26\%20Shoulder\%20Arthroplasty.

14. National Institute of health and welfare. Finnish Arthroplasty register . (2019). https://www.thl.fi/far/\#index.

15. Huang T, Wang W, George D, Mao X, Graves S: What can we learn from Australian Orthopaedic Association National Joint Replacement Registry 2016 annual report?. Ann Joint. 2017, 2: 10.21037/aoj.2017.02.01

16. Ma VY, Chan L, Carruthers KJ: Incidence, prevalence, costs, and impact on disability of common conditions requiring rehabilitation in the United States: stroke, spinal cord injury, traumatic brain injury, multiple sclerosis, osteoarthritis, rheumatoid arthritis, limb loss, and back pain. Arch Phys Med Rehabil. 2014, 95:986-995. 10.1016/j.apmr.2013.10.032

17. Learmonth ID, Young C, Rorabeck C: The operation of the century: total hip replacement . Lancet. 2007, 370:1508-1519. 10.1016/S0140-6736(07)60457-7

18. Siciliani L, Moran V, Borowitz M: Measuring and comparing health care waiting times in OECD countries . Health Policy. 2014, 118:292-303. 10.1016/j.healthpol.2014.08.011

19. Guyen O: Hemiarthroplasty or total hip arthroplasty in recent femoral neck fractures? . Orthop Traumatol Surg. 2019, 105:S95-S101. 10.1016/j.otsr.2018.04.034

20. Sahota O, Currie C: Hip fracture care: all change. Age Ageing. 2008, 37:128-129. 10.1093/ageing/afn007

21. Khan A, Rafferty M, Daurka J: Hemiarthroplasty compared with total hip arthroplasty in fractured neck of femur: a shift in national practice?. Ann R Coll Surg Engl. 2019, 101:86-92. 10.1308/rcsann.2018.0123

22. Dinamarca-Montecinos JL, Améstica-Lazcano G, Rubio-Herrera R, Carrasco-Buvinic A, Vásquez A: Características epidemiológicas y clínicas de las fracturas de cadera en adultos mayores en un hospital público chileno [Article in Spanish]. Rev Méd Chile. 2015, 143:1552-1559. 10.4067/S003498872015001200008

23. Harris IA, Cuthbert A, de Steiger R, Lewis P, Graves SE: Practice variation in total hip arthroplasty versus 


\section{Cureus}

hemiarthroplasty for treatment of fractured neck of femur in Australia. Bone Joint J. 2019, 101-B:92-95.

10.1302/0301-620X.101B1.BJJ-2018-0666.R1

24. Hochfelder JP, Khatib ON, Glait SA, Slover JD: Femoral neck fractures in New York State. Is the rate of THA increasing, and do race or payer influence decision making?. J. Orthop Trauma. 2014, 28:422-426.

10.1097/BOT.0000000000000037

25. Hughes RE, Batra A, Hallstrom BR: Arthroplasty registries around the world: valuable sources of hip implant revision risk data. Curr Rev Musculoskelet Med. 2017, 10:240-252. 10.1007/s12178-017-9408-5 